\title{
Ultrasound Needle Guidance System for Precision Vaccinations and Drug Deliver
}

\author{
Semih Aslan', Mahbubur Rahman'1, Sourav Das', Bonnie Schnitta² \\ ${ }^{1}$ Ingram School Engineering, Texas State University, San Marcos, TX, USA \\ ${ }^{2}$ SoundSense, LLC, Wainscott, NY, USA \\ Email: sa40@txstate.edu, m_r1011@txstate.edu, ssd56@txstate.edu, bonnie@soundsense.com
}

How to cite this paper: Aslan, S., Rahman, M., Das, S., and Schnitta, B. (2021) Ultrasound Needle Guidance System for Precision Vaccinations and Drug Deliver. Circuits and Systems, 12, 1-12. https://doi.org/10.4236/cs.2021.121001

Received: December 15, 2020

Accepted: January 26, 2021

Published: January 29, 2021

Copyright (C) 2021 by author(s) and Scientific Research Publishing Inc. This work is licensed under the Creative Commons Attribution International License (CC BY 4.0).

http://creativecommons.org/licenses/by/4.0/

(c) (i) Open Access

\begin{abstract}
Image-guided needles are currently used for drug delivery in bodies, but the additional time associated with aligning and maintaining the needle's position results in increased patient discomfort or risk of invasion of the human body. In this paper, a needle guidance system using piezoelectric materials is designed and analyzed for precise drug delivery without damaging parts of the body and improving processing time. A piezoelectric generates an ultrasound wave that can propagate through different mediums, and a second piezoelectric crystal can receive that energy and convert it into voltage. A 1D real-time image represents the changes of the voltage induced in the double piezoelectric crystal. Extensive data analysis and visualization are done using different obstacles and location of the needle verified for other mediums. The presence of obstacles in between those crystals can be identified in the real-time grayscale image. The needle can reach its destination using this image information as directional guidance. This guided drug delivery improves patient recovery time and eliminates extra injuries that can be caused due to wrong needle injections, such as lumbar puncture-related nerve damage.
\end{abstract}

\section{Keywords}

Ultrasonic, Needle Guidance System, eSNR, Image Processing, Piezo

\section{Introduction}

Vaccination can be performed on everyone in the world, throughout our lives. These vaccinations are often used as a protective measure against such diseases like Hepatitis B for newborns or to fight off infections as adults aged 65 and older. Sometimes an injection such as platelet-rich plasma (PRP) is needed to recover from acute or tendon injuries, an anesthetic solution is required before 
surgery, or steroid or stem cell injection needs to be accurately located. For these and numerous other injections, the medication's accurate delivery optimizes the efficacy of its application. Physicians who perform these injections often use needles, cannulas, and other sharp objects, which can be invasive to the human body. The injected needle needs to travel through the body, passing nerves, blood vessels, and other internal body organs to reach the minute target area without causing a wound to the patient. In the current image-guided medication process, the practitioner first identifies the region of interest using an ultrasound probe [1]. When the desired anatomy is in view, the practitioner estimates the needle trajectory and insertion point. The inability to perfectly identify the needle tip makes it dangerous to advance the needle, leading to life-threatening seizures [2]. Therefore, the placement of this sharp object must be done with high precision. For this reason, navigated procedures are required to avoid unintended injury. Currently, many of these procedures require more experienced practitioners with the fine motor skill to navigate the needle inside the body safely [2]. Another disadvantage of this current system is that it requires two practitioners to perform the whole procedure: one performing the procedure and assisting with the ultrasound adjustments and medication injection. The requirements of the second practitioner for this task increase cost and limit efficiency. So, making ultrasound system with needle guidance easier to perform medicines in a cost-effective way [3]. Ultrasound with needle guidance is depicted in recent days for medical imagining years due to its lack of harmful effects on the human body. But the detailed analysis of the needle guidance system is still in a pristine state [4]. In this study, we tried to analyze this system mathematically and extract ultrasound waves' behavior from transducers in different mediums and obstacles. Generally, an array of a transducer is used over the skin to generate an ultrasound wave into the body, and it collects the reflected signals. 2D images showing human organs or body parts are developed with the help of the reflected signals. The traditional ultrasound methods of using 2D imaging to find a 3D route lack precision. Even a skilled physician with a high degree of proficiencies, understanding, and synchronization from the clinician, the $2 \mathrm{D}$ guidance does not accurately show the precise location in all three dimensions, which is the end goal of the needle placement. To lessen this impediment, an ultrasound flashlight (USFL) consisting of a needle inserted in a target location of a living body to create an ultrasound image was invented (US Patent: $10,172,588$ B1) [4]. Based on this idea, this paper proposes an experimental setup which can identify the presence and nature of obstacles between two transducers and can show varying grayscale image in real-time.

\section{Background}

Imaging using piezoelectric crystals in a needle has the restriction of needle size that sets the limit of the needle's crystal size. The crystal size limit then sets the frequency used in the imaging. The needle size also establishes the maximum 
array size. With these parameters for imaging, the focus of this research was to establish the mathematical foundation for imaging and the importance of near field imaging. In this paper, only $1 \mathrm{D}$ ultrasound images from a single transducer are demonstrated. Two needles, each consisting of one piezoelectric material and a delivery opening, are used as a transmitter and a receiver. Various metal objects of different sizes are used as obstacles to represent organs in the human body. Grayscale color mapping is used to describe the concentration or the obstacles present between the transducers.

An eSNR pulse-echo system was used that uniquely incorporates the effects of shift-varying impulse responses, spatiotemporal coding, and various beam-formers. Closed-form expressions were found to predict the measured eSNR gains using SMF and STF beamformers, and the gains are significant for an SMF in the near field [5] [6] [7]. In the case of an echo-signal model, a linear equation is considered to define pulse-echo RF signals $g(t)$ in terms of the spatial distribution of an object function $f\left(x, t^{\prime}\right)$ with the spatiotemporal impulse response $h(t)=h(x, t)$ [3] [4] [5] and it is written as,

$$
g(t)=s(t)+e(t)
$$

where, $s(t)=\oint \mathrm{d} x h(x, t) f\left(x, t^{\prime}\right)$.

Here, $s(t)$ defines the RF signals, and $e(t)$ is the noise.

Equation (1) can be transformed from continuous to discrete by sampling the signal in discrete times,

$$
g[l, m]=s[l, m]+e[l, m]
$$

Here, $l$ is described as $0 \leq l \leq L-1$, and $L$ is the range of sampling with interval $\mathrm{T}$ for one $\mathrm{A}$-line in the frame, and $m$ is described as $0 \leq m \leq M-1$, and $M$ defines the number of sequential A lines in the recorded frame. For a stationary scatter, $f\left(x, t^{\prime}\right)=f(x)$ and $t^{\prime}=(l+m L) T$ is the acquisition time of the echo signal. The echo signal to noise (eSNR) is another important term for echo-signal to measure the signal strength over noise [8] [9] [10] within the acquisition time. The eSNR for beamformed RF ultrasound data may be written as,

$$
\mathrm{eSNR}=\mathcal{E}\left\{\sum_{l, m}\left[\mathcal{E}\{g[l, m]\}_{e \mid f}\right]^{2}\right\}_{f} /\left\{\sum \mathcal{E}\left\{e^{2}[l, m]\right\}\right\}_{e}
$$

The notation $\mathcal{E}(g)$ denotes ensemble average of the echo frame $g$ over the noise process $e$ for a fixed realization of stochastic object $f$. For random scattering media, Equation (3) becomes,

$$
\mathrm{eSNR}=\frac{\sigma_{f}^{2}}{\sigma_{e}^{2}}\left(E_{h}\right)
$$

Ignoring the physical order of the signal formation process, the order of the sum and integral can be interchanged to give,

$$
\mathrm{eSNR}=\frac{\sigma_{f}^{2}}{\sigma_{e}^{2}} \oint \mathrm{d} x \phi_{h}(x, 0)
$$


The product of temporal correlation and volume is just the average pulse energy. The shift-invariant resultis,

$$
\operatorname{eSNR}_{r}=\frac{\sigma_{f}^{2}}{\sigma_{e}^{2}} \phi_{h}(x, 0)
$$

A is the reflective amplitude of a single point scatterer, located at $x$ for all $t$. Combining the equations for $\mathrm{eSNR}_{r}$ and $\mathrm{eSNR}_{p}$ yields this relation,

$$
\operatorname{eSNR}_{p}(x)=\frac{A^{2}}{\sigma_{e}^{2}} \phi_{h}^{\prime}
$$

After applying conditions, shift-invariant systems for a single point scatterer yield,

$$
\operatorname{eSNR}_{r}(x)=\frac{\sigma_{f}^{2}}{A^{2}} \oint \mathrm{d} x \phi_{h}^{\prime} \operatorname{eSNR}_{p}(x)
$$

"Spatial filtering" [8] is a term often applied to the processing of RF echo data $\mathrm{g}$ to facilitate beamforming in the process of forming image $\hat{f}$. Spatial filtering effects are observed in the image. We reserve the term "temporal filtering" to refer to compression of coded pulses, and "spatiotemporal filtering" (STF) as a combination of these two methods [9].

The spatial matched filter (SMF) is beamforming filterable to concentrate more of the pulse energy than dynamic receive, focusing on providing a greater $\mathrm{eSNR}_{p}$ [2]. For a point target and matched-filter processing,

$$
\sum_{l, m} \operatorname{eSNR}_{p(m)}^{(i)}(x,[l, m])
$$

The $\operatorname{eSNR}_{r(m)}(x)$ is the eSNR from random scattering media and matchedfilter processing:

$$
\operatorname{eSNR}_{r(m)}=\frac{\sigma_{f}^{2}}{A^{2}} \oint \mathrm{d} x e S N R_{p(m)}(x)
$$

The corresponding eSNR gain for closed region $\Omega$ is,

$$
\gamma_{r(m) \Omega}=\frac{\oint \mathrm{d} x e S N R_{p(m)}(x)}{\oint \mathrm{d} x e S N R_{p}(x)} \geq \min \gamma_{p(m)}(x)
$$

In the above case, the SMF is applied after the RF signals are passed through a beamformer, meaning the echo-signals are filtered after receive-channel summation. Alternatively, an SMF can be applied to signals from each receiver channel before they are added. The latter approach, called ESMF, generates higher lateral resolution and lower sidelobe amplitudes. The disadvantage is that the computational load goes up. The eSNR for the ESMF beamformer is

$$
\operatorname{eSNR}_{e p(m)}(x)=\frac{A^{2} N}{\sigma_{e}^{2}} \frac{\sum_{l, m} Q_{1}^{2}(x,[l, m])}{Q_{1}(x,[0,0])}
$$

The eSNR gain from temporal filtering of coded pulses is,

$$
\gamma_{p(t)}(x)=\frac{\operatorname{eSNR}_{p(t)}(x)}{\operatorname{eSNR}_{p}(x)}
$$


temporal coded excitation enhancement $\gamma_{r(t)}$ and SMF enhancement $\gamma_{r(m)}$. If decoding is successful, the eSNR is increased by K. If it is incomplete, range sidelobes are generated that distort $h(x, t) / \phi_{h}$. Because of this, the expression that follows is an approximation. It is assumed that the pulse compression is complete. The combined gain from spatiotemporal filtering is

$$
\gamma_{r(m, t)}=\gamma_{r(m, t)} \times \gamma_{r(m, t)}=K \frac{\oint{\mathrm{d} x e S N R_{p(m)}(x)}_{\oint \mathrm{d} x e S N R_{p}(x)}}{\oint \sin (x)}
$$

eSNR varies significantly throughout the field for instruments with shift-varying impulse responses. Unfocused near-field regions of a fixed-focus aperture system contain greater eSNRs compared to the focal region and far-field. The greatest gain from the SMF occurs in the far-field. Pulse compression of beamformed signals produces geometric errors in the near field more than in the far-field, thus generating range lobes.

In this experiment, two piezo materials are placed in the air medium, and data was taken from varying distances between two piezo materials. A sinusoidal signal with $10 \mathrm{~V}$ peak-peak and $5 \mathrm{MHz}$ is applied to the transmitter. Different obstacles were placed in between two piezo, and the detailed specification of obstacles is shown in Table 1 . By varying distances and mediums, different obstacles were placed in between the transmitter and receiver, and the amplitude of the received signal was recorded as shown in Table 2 and Table 3, respectively.

\section{Proposed System}

Two piezoneedles are placed face to face at a distance (d), as shown in Figure 1. A sinusoidal signal is applied to a piezoelectric material as a transmitter $(\mathrm{T})$ and received by another piezo as a receiver $(\mathrm{R})$. The experimental laboratory setup for this work is shown in Figure 2. The received signal is then analyzed by MATLAB using an interface, and a decision is made about the medium through an image output. In our experiment, we have used three different mediums such as air, gelatin, and water, by changing the distance from $1 \mathrm{~cm}$ to $6 \mathrm{~cm}$. We labeled the three mediums with three different colors. Black; means highly dense (gelatin), grey; means medium density (water), and white; means a less dense medium (air). Anything between less and highly dense is expressed as a grey. Decision-making about the medium will be discussed later.

Table 1. Dimension of obstacles.

\begin{tabular}{cc}
\hline Obstacle Type & Obstacle Object Size \\
\hline Lead & Diameter: $0.7 \mathrm{~cm}$ \\
Metal & Diameter: $1.5 \mathrm{~cm}$, Height: $6.7 \mathrm{~cm}$ \\
Metal Bar & $12.6 \times 5.2 \times 0.45 \mathrm{~cm}^{3}$ \\
Nut & Día: $0.6 \mathrm{~cm}$ \\
Screw & Diameter: $0.6 \mathrm{~cm}$, Length: $2.6 \mathrm{~cm}$ \\
Knife & $6 \times 1 \times 0.1 \mathrm{~cm}^{3}$ \\
\hline
\end{tabular}


Table 2. Received voltage in air medium varying the obstacles.

\begin{tabular}{|c|c|c|c|}
\hline Obstacle & $\begin{array}{c}\text { Distance } \\
(\mathrm{cm})\end{array}$ & $\begin{array}{c}\text { Vp-p }(\mathrm{mV}) \\
\text { w/o obstacle }\end{array}$ & $\begin{array}{c}\text { Vp-p }(\mathrm{mV}) \\
\text { with obstacle }\end{array}$ \\
\hline \multirow{4}{*}{ Knife } & 2 & 28 & 23.2 \\
\hline & 3 & 23.4 & 19.8 \\
\hline & 4 & 20.2 & 16.6 \\
\hline & 4.4 & 19.2 & 13 \\
\hline \multirow{4}{*}{ Metal Bar } & 2 & 28 & 21 \\
\hline & 3 & 23.4 & 17 \\
\hline & 4 & 20.2 & 15.8 \\
\hline & 4.4 & 19.2 & 12 \\
\hline \multirow{4}{*}{ Screw } & 2 & 28 & 25 \\
\hline & 3 & 23.4 & 20 \\
\hline & 4 & 20.2 & 18.4 \\
\hline & 5.2 & 18 & 17.9 \\
\hline \multirow{4}{*}{ Lead solenoid } & 2 & 28 & 24.5 \\
\hline & 3 & 23.4 & 20.8 \\
\hline & 4 & 20.2 & 18.8 \\
\hline & 5.2 & 18 & 17.4 \\
\hline \multirow{3}{*}{ Nut } & 2 & 28 & 23.8 \\
\hline & 3 & 23.4 & 21 \\
\hline & 4 & 20.2 & 19.2 \\
\hline
\end{tabular}

Table 3. Received voltage in water medium varying the obstacle.

\begin{tabular}{cccc}
\hline Obstacle & $\begin{array}{c}\text { Distance } \\
(\mathrm{cm})\end{array}$ & $\begin{array}{c}\text { Vp-p (mV) } \\
\text { w/o obstacle }\end{array}$ & $\begin{array}{c}\text { Vp-p (mV) } \\
\text { with obstacle }\end{array}$ \\
\hline \multirow{3}{*}{ Knife } & 1.1 & 102.4 & 93 \\
& 2.9 & 98 & 79.2 \\
& 4.4 & 96.8 & 76 \\
\hline \multirow{3}{*}{ Metal Bar } & 2 & 104.8 & 88.8 \\
& 3 & 98 & 81.6 \\
& 4.4 & 96.4 & 86.4 \\
\hline \multirow{3}{*}{ Screw } & 2 & 28 & 25 \\
& 3 & 23.4 & 20 \\
& 4 & 20.2 & 18.4 \\
& 5.2 & 18 & 17.9 \\
\hline \multirow{2}{*}{ Lead solenoid } & 2 & 28 & 24.5 \\
& 3 & 23.4 & 20.8 \\
& 4 & 20.2 & 18.8 \\
& 5.2 & 18 & 17.4 \\
\hline \multirow{2}{*}{ Nut } & 2 & 28 & 23.8 \\
& 3 & 23.4 & 21 \\
& 4 & 20.2 & 19.2 \\
\hline & & & \\
\hline
\end{tabular}




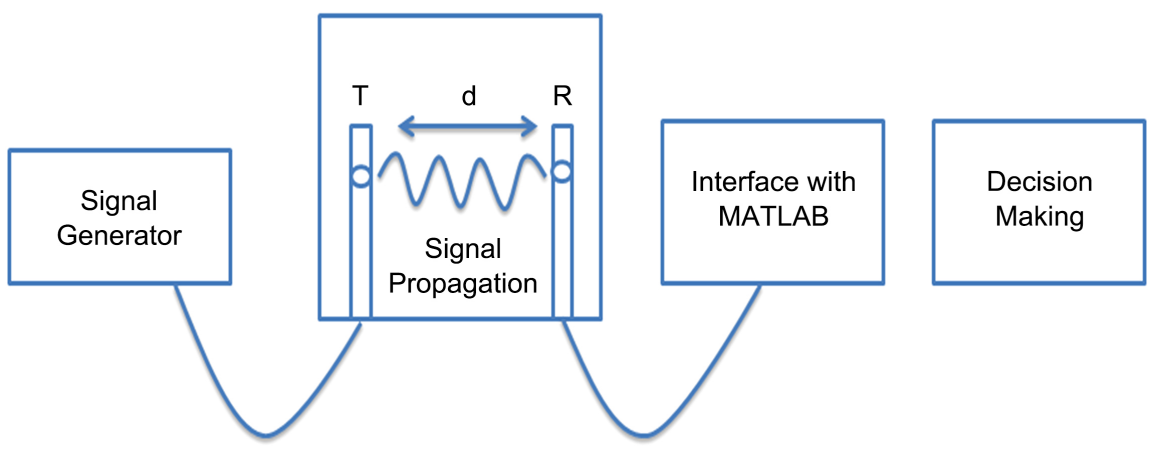

Figure 1. Schematicdiagram of the system.

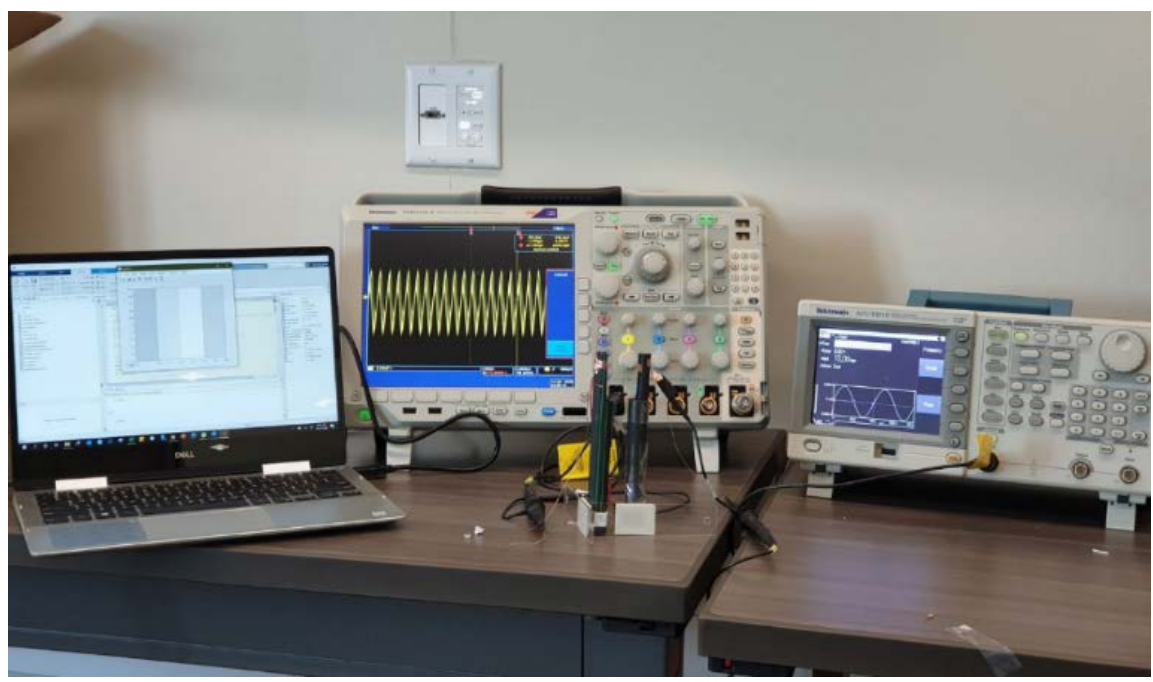

Figure 2. Experimental setup.

The velocity and attenuation of the ultrasound waves are strongly dependent on the medium between the transmitter and receiver. The attenuation coefficient of ultrasound waves at $5 \mathrm{MHz}$ in water medium is $0.0543 \mathrm{~dB} / \mathrm{cm}$, and in air, the medium is $11 \mathrm{~dB} / \mathrm{cm}$ [10]. The amplitude of ultrasound waves decays [11] [12] [13] [14], as shown in Equation (15), with distance and attenuation constant.

$$
A=A_{0} \mathrm{e}^{-\propto z}
$$

where,

$A=$ Amplitude of received signal after the distance;

$A_{0}=$ amplitude of the transmitted signal;

$\alpha=$ attenuation co-efficient.

With the increase of distance, the amplitude of the sound waves decreased. The attenuation coefficient depends on the medium. A higher attenuation constant means higher loss, so less energy can be received. As water has a lower attenuation constant than air, the received signal will be higher in amplitude in a water medium than in air. As we were operating with a higher frequency, fluctuation in the amplitude raised an issue. Accordingly, we used a linear equation and mapped the received signal with a grayscale [0 - 255] image. 


\section{Results}

Six different sizes of obstacles were used to analyze the effect on signal attenuation. Along with obstacles, the effects of mediums were also investigated. Different mediums have different densities and attenuation coefficients which affect the signal propagation.

In Figure 3, data were taken in the air medium with and without an obstacle (a knife) between the transmitter and receiver. Due to the presence of the knife, the received signal in the receiver decreases. This data was fitted by polynomial regression.

Again, both the transmitter and receiver were sunk into the water in Figure 4. The same procedure as the air was also done for a water medium. With or without obstacles, the water medium gave a higher amplitude than the air medium, and this is evident in Equation (15). Although the piezo materials were moved in the water, there were still some air pockets between piezo materials and water to keep the piezo materials dry. This was reviewed for its effect on the results. It was confirmed to be consistent and, therefore, minor. That is, once these air pockets are removed with the final USFL, the mathematical foundation that in turn will result in the imaging will remain valid and robust.

Figure 5 provides a comparison between the water and air mediums. Signal amplitude is higher in the water medium, as the velocity of ultrasound waves is higher in a denser medium. The attenuation of the signal increases with the increasing gap, and two curves show almost the same trend with an increasing gap between two piezo.

The directivity of the piezo was analyzed by placing two piezo at different angles. Figure 6 denotes the omnidirectionally of the piezo materials, which may create interference problems in the case of a piezo array.

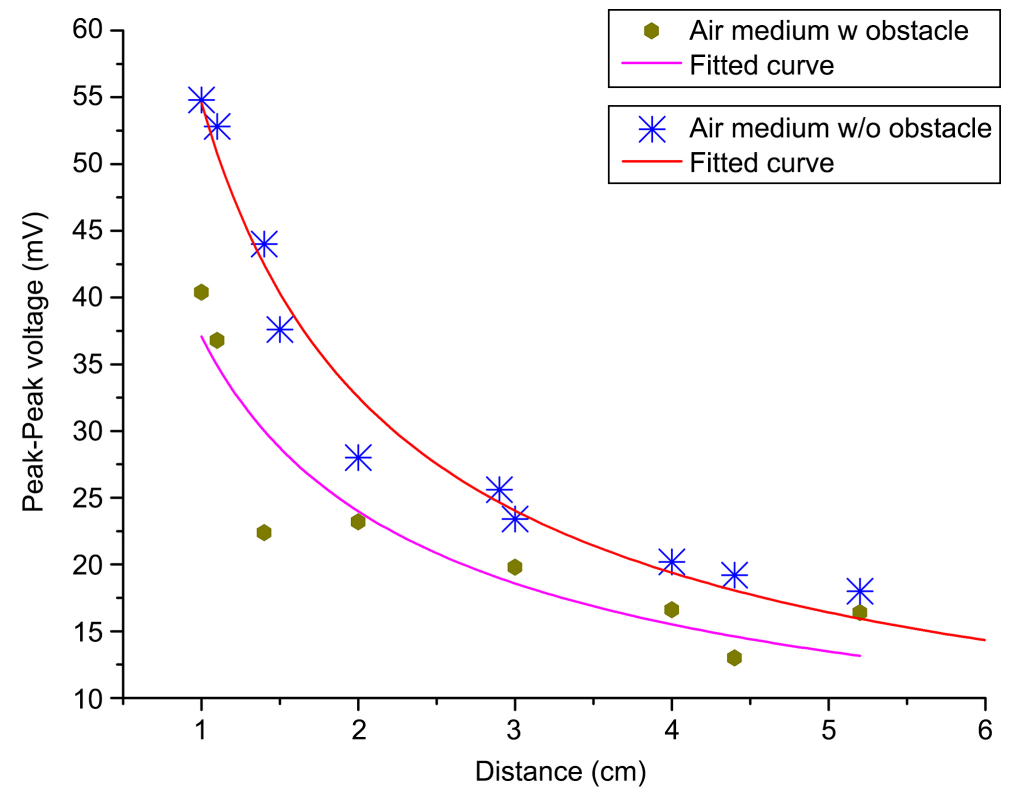

Figure 3. Voltage variations with distance with \& without obstacle (knife). 


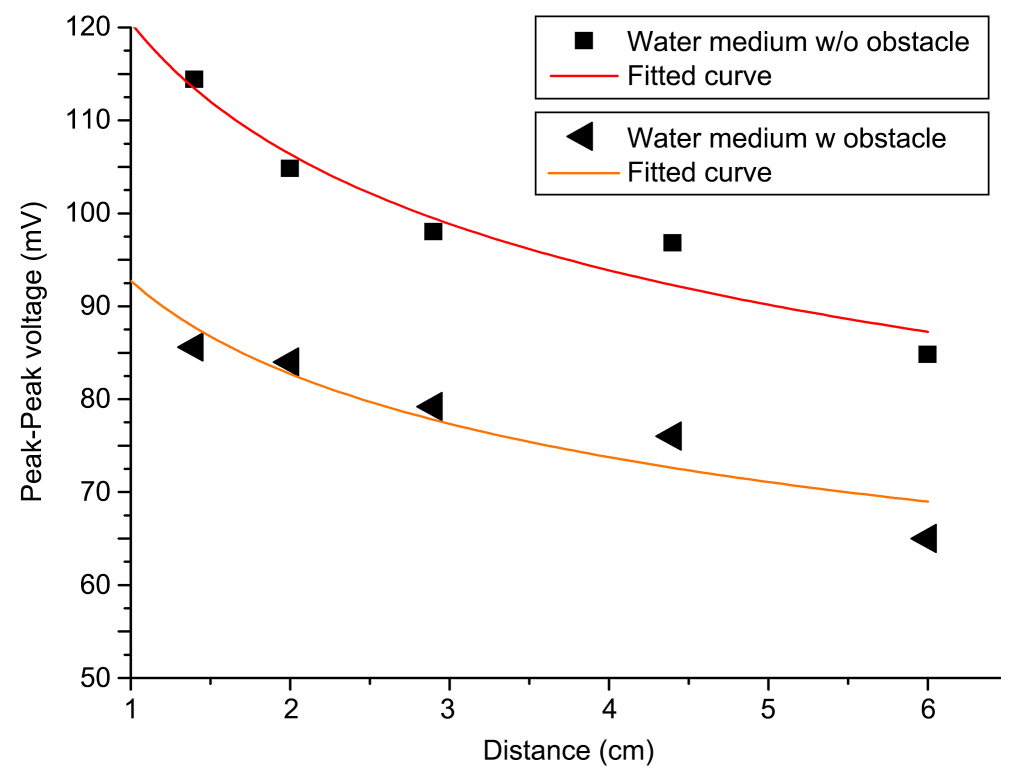

Figure 4. Voltage variations with \& without obstacle (knife) in water.

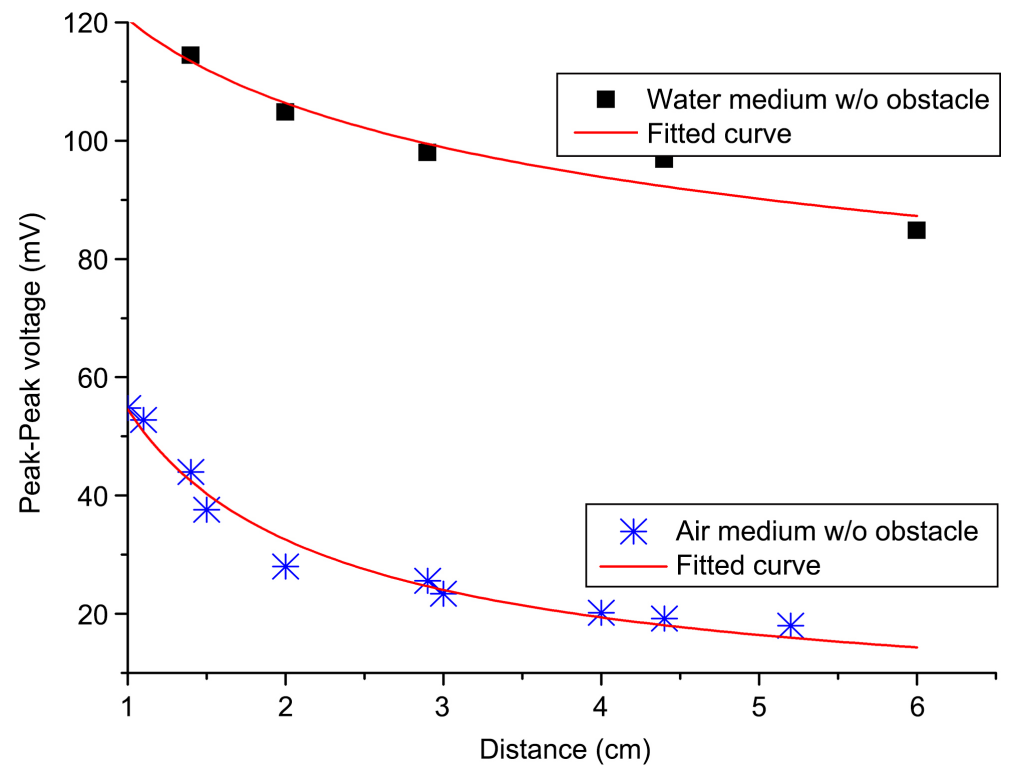

Figure 5. Voltage variations with distance varying medium.

After collecting these values in different mediums, distances, and angles, we fixed two extreme points (upper limit and lower limit) between which the amplitude of the signal will fluctuate depending upon the mediums and distances. The peak-peak voltage of the received signal in the water medium varied from $120 \mathrm{mV}$ to $90 \mathrm{mV}$, whereas in the air medium, it varied from $58 \mathrm{mV}$ to $20 \mathrm{mV}$ with a gap from $1 \mathrm{~cm}$ to $6 \mathrm{~cm}$ (w/o obstacle). We then applied a linear correlation between these received values and a grayscale [0 - 255], as seen in Figure 7. When the receiving voltage is increased, the color should be biased to the black region of grayscale, and if it is decreased, the color should change to white. MATLAB was used to process the data in real-time. 


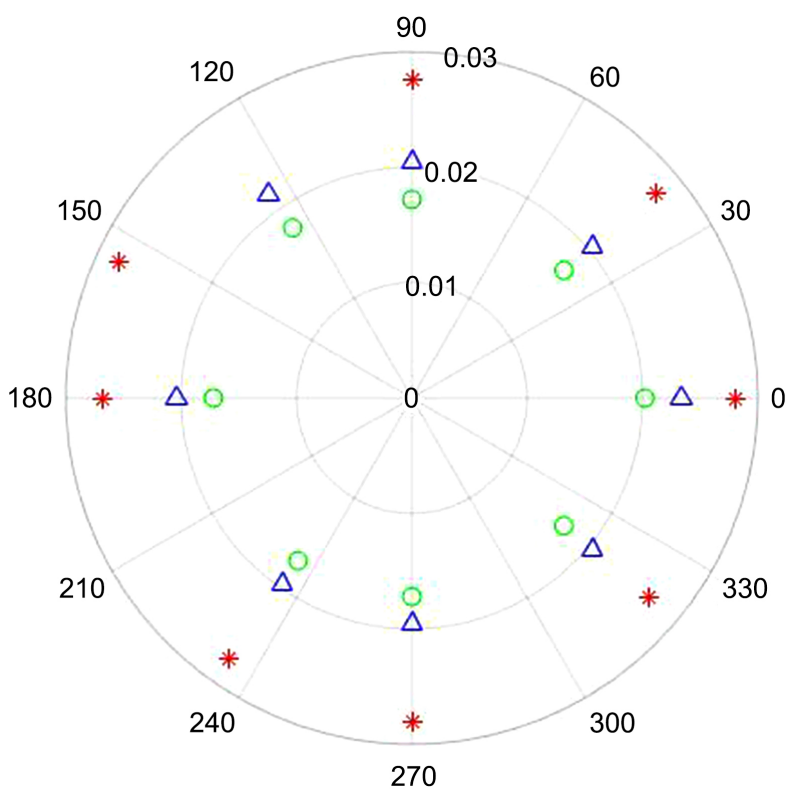

* distance: $2 \mathrm{~cm}$

$\triangle$ distance: $3 \mathrm{~cm}$

distance: $4 \mathrm{~cm}$

Figure 6. Horizontal $360^{\circ}$ received voltage for different distances.

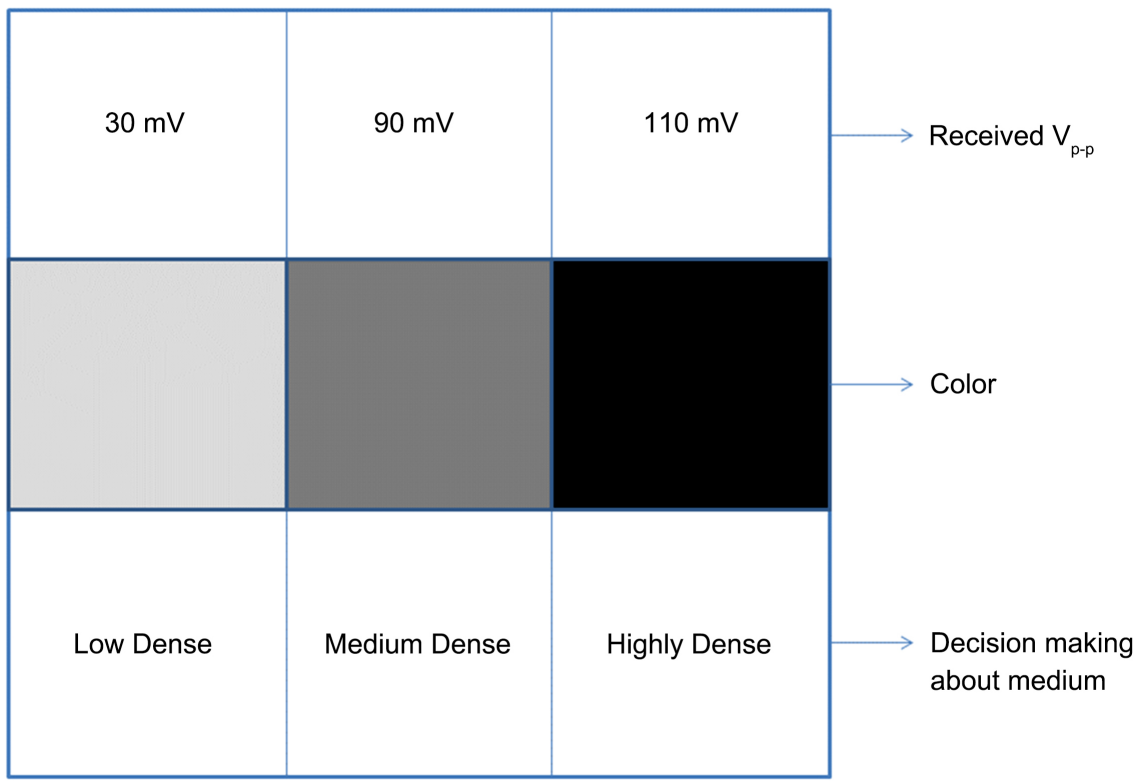

Figure 7. Representation of medium in grayscale by received voltages.

\section{Conclusion}

The real-time 1D image representation is the first step of a needle guidance system using a piezoelectric crystal. It showed the efficacy of the mathematical approach for near field imaging. Unlike the other existing conventional ultrasound and passive magnetic ultrasound needle guidance technology, our experiment was based on different low power and low-cost materials which can be easily constructed. Our proposed system can detect any obstacle that will be implemented as other organs and the associated density differential of the human body. This model also provides necessary information on the depth and size of 
the obstacle which the needle encounters. Using the array of piezo crystals, the pictorial system's precision could be further enhanced, and consequently, contrast resolution can be affected. Also, future work includes 2D and 3D data representation, machine learning-based data analysis, and RGB color representation of the needle location using different mediums. Final work will include real-time field experiments and data collections and analysis.

\section{Acknowledgements}

We would like to thank SoundSense NYC, NY, Robert J Hackworth, MD Solaris Anesthesia ServicesImperial Beach, CA, and Carter Sigmon MD, MHA Fairbanks Medical GroupRancho Santa Fe, California, for their support.

\section{Conflicts of Interest}

The authors declare no conflicts of interest regarding the publication of this paper.

\section{References}

[1] Brattain, L.J., Floryan, C., Hauser, O.P., Nguyen, M., Yong, R.J., Kesner, S.B., Corn, S.B. and Walsh, C.J. (2011) Simple and Effective Ultrasound Needle Guidance System. Annual International Conference of the IEEE Engineering in Medicine and Biology Society, 2011, 8090-8093. https://doi.org/10.1109/IEMBS.2011.6091995

[2] Beach, M.L., et al. (2009) A Needle Guide Device is Better than a Free Hand Technique for Ultrasound Guided Cannulation of the Internal Jugular Vein. The Internet Journal of Medical Simulation, 2, 11.

[3] Royer, T. (2001) Nurse-Driven Interventional Technology: A Cost and Benefit Perspective. Journal of Infusion Nursing, 24, 326-331. https://doi.org/10.1097/00129804-200109000-00007

[4] Sigmon, C.H., Hackworth, J. and Schnitta, B.S. (2019) Ultrasound Guidance system and Method. United States Patent No. 10172588

[5] Liu, J., Kim, K. and Insana, M.F. (2007) SNR Comparisons of Beamforming Strategies. IEEE Transactions on Ultrasonics, Ferroelectrics, and Frequency Control, 54, 1010-1017. https://doi.org/10.1109/TUFFC.2007.346

[6] Tupholme, G.E. (1969) Generation of Acoustic Pulses by Baffled Plane Pistons. Mathematika, 16, 209-224. https://doi.org/10.1112/S0025579300008184

[7] Stepanishen, P.R. (1981) Acoustic Transients from Planar Axisymmetric Vibrators Using the Impulse Response Approach. The Journal of the Acoustical Society of America, 70, 1176-1181. https://doi.org/10.1121/1.386949

[8] Abbey, C.K., Zhu, Y., Bahramian, S. and Insana, M.F. (2017) Linear System Models for Ultrasonic Imaging: Intensity Signal Statistics. IEEE Transactions on Ultrasonics, Ferroelectrics, and Frequency Control, 64, 669-678. https://doi.org/10.1109/TUFFC.2017.2652451

[9] Tobergte D.R. and Curtis, S. (2013) Detection, Estimation, and Modulation Theory. Journal of Chemical Information and Modeling, 53, 1689-1699.

[10] Liu, J. and Insana, M.F. (2005) Coded Pulse Excitation for Ultrasonic Strain Imaging. IEEE Transactions on Ultrasonics, Ferroelectrics, and Frequency Control, 52, 
231-240. https://doi.org/10.1109/TUFFC.2005.1406549

[11] Liu, J., Kim, K., Insana, M.F. and Brunke, S. (2005) Beamforming Using Spatio-Temporal Filtering. IEEE Ultrasonics Symposium, 2, 1216-1219.

[12] Fitzhugh, S.M., Gibson, C.B., Spiro, E.S. and Butts, C.T. (2016) Spatio-Temporal Filtering Techniques for the Detection of Disaster-Related Communication. Social Science Research, 59, 137-154. https://doi.org/10.1016/j.ssresearch.2016.04.023

[13] Szilard, J. (1978) Ultrasonic testing of materials. Ultrasonics, 16, 187. https://doi.org/10.1016/0041-624X(78)90077-X

[14] Lukanova, D.V., Nikolov, N.K., Genova, K.Z., Stankev, M.D. and Georgieva, E.V. (2015) The Accuracy of Noninvasive Imaging Techniques in Diagnosis of Carotid Plaque Morphology. Open Access Macedonian Journal of Medical Sciences, 3, 224-230. 